\title{
Prostate Cancer Chemoprevention Targeting High Risk Populations: Model for Trial Design and Outcome Measures
}

Nagi Kumar ${ }^{1,2 *}$, Theresa Crocker ${ }^{1}$, Tiffany Smith ${ }^{1}$, Julio Pow-Sang ${ }^{1,2}$, Philippe E. Spiess ${ }^{1,2}$, Shanjayla Connors ${ }^{1}$, Ganna Chornukur ${ }^{1}$, Shohreh Iravani Dickinson ${ }^{1,2}$, Wenlong Bai ${ }^{3 *}$, Christopher R. Williams ${ }^{4}$, Raoul Salup ${ }^{5}$ and Wui Fu ${ }^{6}$

${ }^{1}$ Department of Epidemiology, H. Lee Moffitt Cancer Center \& Research Institute, University of South Florida, College of Medicine, Tampa, Florida ${ }^{2}$ Department of Oncological Sciences, University of South Florida, College of Medicine, Tampa, Florida

${ }^{3}$ Department of Pathology and Cell Biology, H. Lee Moffitt Cancer Center \& Research Institute, University of South Florida, College of Medicine, Tampa, Florida ${ }^{4}$ Department of Surgery, University of Florida, College of Medicine, Jacksonville, Florida

${ }^{5}$ Department of Urology, James A Haley Veterans Administration Hospital, University of South Florida, College of Medicine, Tampa, Florida

${ }^{6}$ Department of Cellular Biology, University of South Florida, College of Medicine, Tampa, Florida

\begin{abstract}
Inspite of the large number of promising nutrient-derived agents demonstrating promise as potential chemopreventive agents, most have failed to prove effectiveness in clinical trials. Critical requirements for moving nutrient-derived agents to recommendation for clinical use include adopting a systematic, molecular-mechanism based approach and utilizing the same ethical and rigorous methods such as are used to evaluate other pharmacological agents. Preliminary data on a mechanistic rationale for chemoprevention activity as observed from epidemiological, in vitro and preclinical studies, phase I data of safety in high-risk cohorts are required to inform design of phase II clinical trials. Additionally, a valid panel of biomarkers representing the hypothesized carcinogenesis pathway for measuring efficacy must be utilized to evaluate effectiveness in these trials. The goal of this paper is to provide a model, using a systematic approach for evaluating the safety, effectiveness and mechanism of action of a well characterized nutrient-derived agent- isoflavones - in a phase II clinical trial for prostate cancer (CaP) chemoprevention, targeting a population of African American (AA) and Caucasian men. Based on our previous observations, we hypothesize that the effects of isoflavones on prostate carcinogenesis are mainly mediated through the down regulation of androgen receptor (AR) and AR activity in AA men is higher due to its shorter length of Glutamine repeats in its $\mathrm{N}$-terminus. We thus believe that isoflavones will exert a stronger protective effect for $\mathrm{CaP}$ in AA men and cause a higher activation of FOXO factors and their target genes. The aim of the study is to evaluate the comparative effectiveness of the study agent and placebo, in addition to a comparison of the effectiveness and safety in African American men compared to Caucasian men treated with this agent.
\end{abstract}

Keywords: African American (AA); Androgen Receptor (AR); Isoflavones; Carcinogenesis; Chemoprevention; Caucasian men

\section{The Disease: Prostate Cancer an Unequal Burden in African American (AA) Men}

The American Cancer Society estimates that there will be about 240,890 new cases of prostate cancer (CaP) in the United States in 2011 and about 33,720 men will die of this disease [1]. The initiation and progression of $\mathrm{CaP}$ is a multi-step process including several intermediate steps that may involve a complex series of both exogenous and endogenous factors [2-4]. Although it is clear that clinical $\mathrm{CaP}$ incidence and mortality vary greatly between populations, the frequency of latent $\mathrm{CaP}$ is evenly distributed among populations, suggesting that external factors such as diet and other lifestyle factors are important in the transformation from latent into more aggressive, clinical cancer [2-4]. An estimated 35, 110 cases of prostate cancer are expected to occur among African American men in 2011, accounting for $40 \%$ of all cancers diagnosed in AA men. Between 2003 and 2007, the average annual $\mathrm{CaP}$ rate was $60 \%$ higher in AA men compared to white men [1]. In addition; AA men have the highest mortality rate of any other racial or ethnic group in the US. Although the overall incidence of and mortality from $\mathrm{CaP}$ has been declining in Caucasian men since 1991, possibly due to improved diagnostic techniques, better screening and improved surgical and radiologic treatments, the decline in AA men lags behind Caucasian men. For AA men with a family history of hereditary $\mathrm{CaP}$, the increased risk is even greater [1]. Autopsy studies and clinical findings support the argument that
CaP exhibits more aggressive biological behavior in AA men than that observed in other populations.

To successfully resolve this health disparity in prostate cancer incidence, mortality and morbidity, it is critical to focus on addressing the need to eliminate the disparity in risk and incidence of prostate cancer in the African-American population. In addition to early screening and detection, high-risk AA men are prime targets for chemoprevention strategies. Chemoprevention refers to the inhibition of preinvasive and invasive cancer and its progression or treatments

${ }^{*}$ Corresponding authors: Nagi B. Kumar, PhD, RD, FADA, Senior Member, Population Sciences Division, Director, Cancer Chemoprevention, Moffitt Cancer Center, Professor, Oncologic Sciences, University of South Florida College of Medicine, 12902 Magnolia Drive Tampa, FL 33612, USA, Tel: 8137456885; Fax: 8137457183; E-mail: nagi.kumar@moffitt.org

Wenlong Bai, PhD, Professor, Department of Pathology and Cell Biology, USF College of Medicine, Member, Molecular Oncology Program, H. Lee Moffitt Cance Center, 12901 Bruce B. Downs Blvd., MDC 11, Tampa, FL 33612-4799, USA, Tel: 813-974-0563; Fax: 813-974-5536; E-mail: wbai@health.usf.edu

Received December 09, 2011; Accepted January 08, 2012; Published January 10, 2012

Citation: Kumar N, Crocker T, Smith T, Pow-Sang J, Spiess PE, et al. (2012) Prostate Cancer Chemoprevention Targeting High Risk Populations: Model for Trial Design and Outcome Measures. J Cancer Sci Ther S3:007. doi:10.4172/19485956.S3-007

Copyright: (C) 2012 Kumar N, et al. This is an open-access article distributed under the terms of the Creative Commons Attribution License, which permits unrestricted use, distribution, and reproduction in any medium, provided the original author and source are credited. 
of identifiable precancers [4]. Chemoprevention efforts require a thorough understanding of the mechanism of carcinogenesis including signaling and metabolic pathways and genetic progression pathways. New technologies in genomics and proteomics have spurred this field of research. The use of this knowledge to develop pharmacologic agents (including nutrient-derived) to reverse or halt the process of carcinogenesis is called chemoprevention. Agents for chemoprevention include anti-promotion and anti-progression agents that prevent the growth and survival of cells that are already committed to become malignant [4]. Several nutrient-derived agents have demonstrated promise as potential chemopreventive agents in the prevention of prostate cancer. The experience with recruitment of minorities in the SELECT trial and our surveys in men at high risk for prostate cancer, including AA men has clearly demonstrated that AA men are eager and willing to participate in chemoprevention trials $[5,6]$. This is more so in AA males who are higher risk for the disease or who are already diagnosed with prostate cancer. These cohorts are thus ideal target populations (men at high risk or diagnosed with localized $\mathrm{CaP}$ ) with a treatment time period (surveillance, watchful-waiting period or presurgical) which provides the best opportunity for evaluating promising agents for chemoprevention and delineating the mechanism of action and molecular targets of specific promising agents to prevent disease progression, shifting the time from diagnosis to invasive disease. The goal of this paper is to provide a model for evaluating the comparative effectiveness of a well characterized agent - isoflavones - in a phase II clinical trial for prostate cancer chemoprevention targeting a population of African American and Caucasian men.

\section{Promising Agent for Chemoprevention}

\section{Evidence from epidemiological studies}

Epidemiological and laboratory studies have demonstrated that several nutrients, including isoflavones, could induce apoptosis, suppressing the formation and growth of human cancers including $\mathrm{CaP}$ [7-12]. Epidemiological studies have consistently reported lower incidence of clinically evident disease in populations consuming isoflavones. An inverse relationship between dietary intake, plasma [7-12] and prostatic fluid [11] concentrations of isoflavones and the incidence of $\mathrm{CaP}$ and benign prostatic hyperplasia (BPH) has been observed in these populations, demonstrating the potential role of isoflavones in mediating epigenetic effects. Thus the potential preventive properties of isoflavones in $\mathrm{CaP}$, as demonstrated by evidence from epidemiological studies although limited, appear promising.

\section{Evidence from in vitro studies}

In vitro data have consistently shown that genistein modulates cell proliferation [13-17], angiogenesis [18,19], tumor cell invasion and tumor metastasis [13,20-21], cell cycle regulation [22], antioxidant $[20,23]$ and induction of apoptotic cell death [24], indicating that purified isoflavones are promising chemopreventive agents, with several cellular effects which are both genomic and non-genomic. However, to date, the molecular mechanism for cancer-preventive effects of isoflavones is poorly understood.

Our computational docking and in vitro and in vivo proteasome activity studies confirmed that indeed the isoflavone genistein, similar to EGCG is a proteasome inhibito $[25,26]$. We also found that genistein at $1 \mu \mathrm{M}$ could inhibit $\sim 30 \%$ of the chymotrypsin-like activity of purified $20 \mathrm{~S}$ proteasome. It has been reported that plasma levels of genistein are in a range of $0.5-2.5 \mu \mathrm{M}$ and the concentrations of genistein also vary in different tissues and organs. It is therefore possible that a partial inhibition of the proteasome activity by genistein at a physiological concentration might contribute to its reported cancer-preventative effects.

Among different soy compounds, genistein was the most potent inhibitor of the proteasomal chymotrypsin-like activity, which was consistent with the previous reports where it has also been shown that genistein is the most potent soy isoflavone. Inhibition of proteasome activity by genistein in prostate cancer cells (LNCaP) was associated with increased levels of $\mathrm{p} 27^{\mathrm{Kip} 1}$, IkB- $\alpha$, an important inhibitor of the tumor survival factor $\mathrm{NF \kappa B}, \mathrm{Bax}$, and ubiqutinylated proteins, accompanied by induction of apoptotic cell death. We also found that genistein was the most potent one among all the tested isoflavones to induce Bax accumulation and PARP cleavage. However, daidzein and glycetin, in addition to genistein, were able to accumulate $\mathrm{p} 27^{\mathrm{Kip} 1}$ protein. These results suggest that accumulation of Bax and IkB- $\alpha$ is associated with apoptosis induction while p27 $7^{\text {Kip1 }}$ accumulation is probably associated with $G_{1}$ arrest [24].

Although many activities of genistein have been documented in literature, the primary molecular target for genistein remains to be identified. In this regard, it is important to note that genistein is also considered a phytoestrogen, owing to its structural and functional similarity to estrogens. Various studies showed that genistein binds to ERs and exhibits receptor- and cell-specific agonistic and agonistic activity on estrogen receptors, ER $\alpha$ and ER $\beta$, in a way very similar to tamoxifen, which is a selective estrogen receptor modulator (SERM). Thus, it appears that ERs represent potential primary molecular targets for genistein.

FOXO proteins are a family of forkhead transcriptional factors, which are characterized by a conserved DNA binding domain termed the "Forkhead box" [27]. Mammalian FOXO factors include FOXO1 (previously known as FKHR), FOXO3A (previously known as FKHRL1), FOXO4 (previously known as AFX) and FOXO6 [28]. These factors control the expression of a variety of genes that regulate essential cellular processes (Figure 1), such as cell cycle, apoptosis, oxidative stress, atrophy, energy homeostasis and glucose metabolism, tumorigenesis and the detoxification of reactive oxygen species (ROS) and the control of stem cell lifespan. Our studies [29] showed that androgens and estrogens repressed the FOXO1 activity in prostate

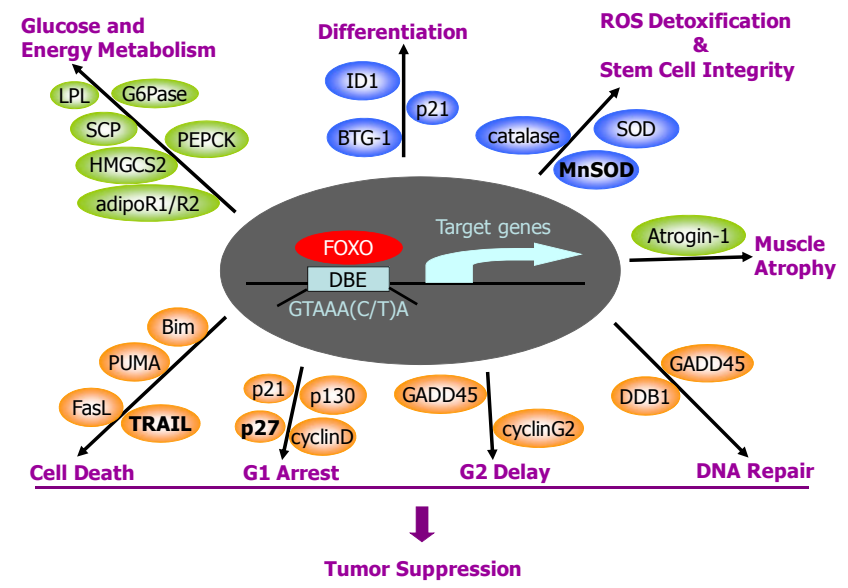

Figure 1: FOXO target genes and the role of FOXO factors in essentia cellular processes. The picture was adapted from Greer and Brunet (2005) Oncogene 24: 7410-7425. 
Citation: Kumar N, Crocker T, Smith T, Pow-Sang J, Spiess PE, et al. (2012) Prostate Cancer Chemoprevention Targeting High Risk Populations: Model for Trial Design and Outcome Measures. J Cancer Sci Ther S3:007. doi:10.4172/1948-5956.S3-007

cancer cells, a process that is independent of the PKB/AKT-mediated FOXO1 phosphorylation. The repression was androgen receptor (AR) and ERa-dependent and mediated through the formation of receptorFOXO1 protein complex. Later, an independent study showed that androgens decreased the level of FOXO1 protein expression in prostate cancer cells [30]. More importantly, it has been reported that genistein down regulates AR expression in LNCaP prostate cancer cells at both mRNA and protein level [31]. More interestingly, the AR down regulation was blocked by $17 \beta$-estradiol, suggesting that the down regulation of the AR is mediated through one of the ERs. Since LNCaP cells express mainly ER $\beta$, it is reasonable to expect that $\mathrm{ER} \beta$ is the receptor that mediates the down regulation of AR by genistein. As shown in Figure 2, we reproduced the published data that genistein down regulated the $\mathrm{AR}$ in a dose dependent manner and that the down regulation is relieved by $17 \beta$-estradiol at $1 \mu \mathrm{M}$. However, when the relief experiment was performed with $10 \mu \mathrm{M} 17 \beta$-estradiol, it did not work. This dosagedependency suggests that the estrogen relief is most likely due to the competition between genistein and $17 \beta$-estradiol for the binding to $\operatorname{ER} \beta$ in LNCaP cells. Consistent with the down regulation of AR expression, pretreatment of LNCaP cells with genistein relieved the androgen induced inhibition of FOXO1 in a dosage dependent manner (Figure 3) as well as the androgen protection of LNCaP cells from FOXO1-induced apoptosis (Figure 4).

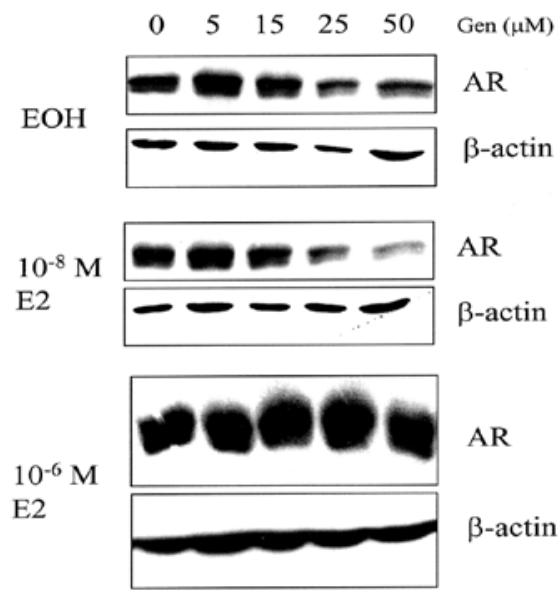

Figure 2: Genistein down regulates the level of AR expression, which is relieved by high level of $17 \beta$-estradiol. $L N C a P$ cells were pretreated with $\mathrm{EOH}$ or E2 for $12 \mathrm{hrs}$ and then with genistein (Gen) at indicated concentrations for $24 \mathrm{hrs}$ and AR level was determined with anti-AR antibody.

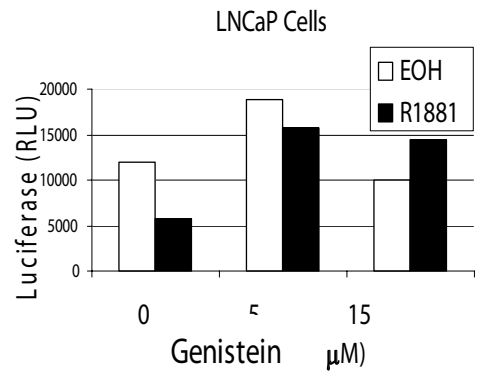

Figure 3: Genistein suppresses androgen-induced inhibition of FOXO1 activity. LNCaP cells were transfected FOXO1 and reporter and treated with genistein (Gen) at indicated concentrations for $24 \mathrm{hrs}$ followed by treatment with $\mathrm{EOH}$ or R1881 for another 24 hrs before FOXO1 activity was measured.

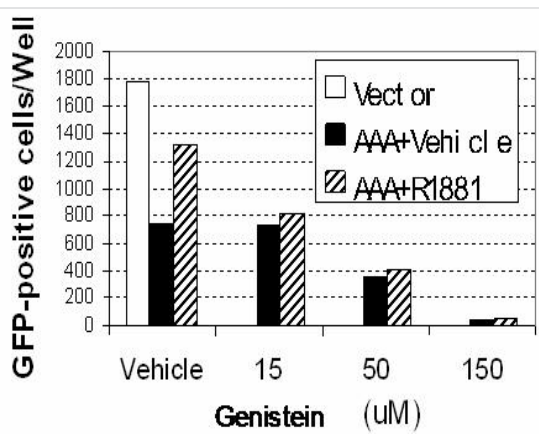

Figure 4: Genistein suppresses androgen-mediated protection of FOXO1induced cell death in LNCaP cells. Cells were transfected with $0.5 \mu \mathrm{g}$ GFP vector (pLNCE), and $0.1 \mu \mathrm{g}$ FOXO1:TSS (AAA) and treated with genistein with or without R1881 for 24 hrs. The viability of transfected cells in each well was determined by counting the total number of green cells.

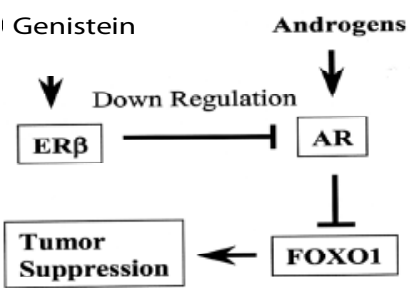

Figure 5: Our working model depicting ER $\beta$ as the primary molecular target for genistein and FOXO factors as the novel nuclear mediators for genistein action in prostate cancer cells.

Overall, the studies summarized above suggest that ER $\beta$ may be the primary molecular target for genistein and that through ER $\beta$, genistein increases FOXO1 activity as well as its level of expression in prostate cancer cells through the down regulation of the AR (Figure 5). Our studies identify FOXO factors as novel nuclear factors that mediate the tumor suppressive effect of genistein in prostate cancer cells and the apoptosis and cell cycle arrest mediated through FOXO factors as novel molecular pathways for genistein action in prostate cancer cells. Because we hypothesize that the effect of genistein is mainly mediated through the down regulation of AR and AR activity in AA men is higher due to its shorter length of Glutamine repeats in its N-terminus [32], we believe genistein will exert a stronger protective effect for prostate cancer in AA men and cause a higher activation of FOXO factors and their target genes.

\section{Evidence from clinical trials}

Phase I trials have demonstrated the clinical characteristics and pharmacokinetics and safety of whole soy and purified isoflavones with single and multiple-dose administration in healthy, early stage or treated cancer patient cohorts [28,33-34] with doses of purified soy isoflavones ranging from [33] 1-16 mg/kg body weight, where some of the doses were higher than those previously administered to humans as whole soy proteins, without significant clinical toxicity. A few pilot Phase II clinical trials including our study, have demonstrated a trend towards stabilization or reduction of PSA with short-term isoflavone supplementation in $\mathrm{CaP}$ patient populations, without significant clinical toxicity [34-40] with the exception of GI symptoms.

In our first Phase II clinical trial of isoflavone supplementation in men with localized $\mathrm{CaP}$ [38], we administered whole soy isoflavones at a dose of $60 \mathrm{mg}$ in 60 grams soy protein. Fifty-nine patients completed 
the 12 -week intervention. Serum free testosterone was reduced or showed no change in $61 \%$ of subjects in the isoflavone group compared to $33 \%$ in the placebo group. Serum total PSA decreased or was unchanged in $69 \%$ of the subjects in the isoflavone treated group compared to $55 \%$ in the placebo group and nineteen percent of subjects receiving soy isoflavones reduced total PSA by two points or more during the intervention period. Seventeen (17) subjects were unable to complete the study reporting constipation and GI symptoms such as bloating, discomfort, diarrhea and pain in both the groups, which were attributable to the protein content of these supplements. Since the potent agent in these soy compounds that have the significant effect on prostate carcinogenesis are isoflavones and not the protein, and as demonstrated by these earlier trials, have few clinical symptoms attributable to them, purified isoflavones preparations may be the ideal choice of agent in clinical trials.

Based on our experience and the results of these earlier Phase I and II studies, we then hypothesized that supplementation with a constant dose of purified isoflavones ( $v s$. a placebo) will produce an increase in plasma levels of isoflavones which will be correlated with stabilization or reduction in surrogate markers of proliferation (serum total PSA) and thereby contribute to a decrease or stabilization of disease progression in men diagnosed with early stage CaP. To test this hypothesis, we completed a pilot Phase II randomized, doubleblinded, placebo-controlled trial [8] of men with early stage $\mathrm{CaP}$ (Gleason 2-6) to receive purified isoflavones, (Prevastein $\mathrm{HC}^{\circledR} 80 \mathrm{mg} /$ day, IND \#61,949 Kumar) vs. a placebo, and observed the effectiveness of the study agent in producing an increase in plasma levels of isoflavones (daidzein, glycitein and genistein) and a corresponding reduction/stabilization in serum total PSA. In addition, our aim was to evaluate compliance to study agents and related toxicity. In this Phase II trial, evaluation of the effectiveness of intervention was based on the magnitude of change in plasma levels of isoflavones in the isoflavone-supplemented group compared to the placebo group and a corresponding stabilization or reduction in surrogate markers of proliferation (total PSA), increase in serum estradiol and reduction in free testosterone that could be achieved. Fifty subjects completed the 12-week intervention. Significant increases in plasma isoflavones $(\mathrm{P}=<0.001)$ were observed from baseline to 4 and 12 weeks in the isoflavone-treated group compared to placebo, without producing clinical toxicity [39]. Although greater mean reduction of serum free testosterone was observed in subjects in the isoflavone-treated group compared to the placebo group, these changes were not statistically significant for this duration of intervention $(P=0.3)$ [34]. Increasing concentrations of plasma isoflavones diadzein $(\mathrm{P}=0.02)$ and genistein $(\mathrm{P}=0.01)$ in the isoflavone-treated group were inversely correlated to changes in serum PSA compared to the placebo arm [34].

In another Phase II randomized-controlled trial [41] to evaluate the safe and effective dose of isoflavones to be used in future clinical trials for prostate cancer prevention; forty-five eligible men were supplemented with 40,60 and $80 \mathrm{mg}$ of purified isoflavones or no supplement from biopsy to prostatectomy. Compliance to study agent, toxicity, changes in plasma isoflavones, serum steroid hormones, prostate specific antigen (PSA) and tissue Ki-67 were analyzed from baseline to completion of study. Forty-four subjects completed the study with duration of intervention of $30(+/-3)$ days. We observed significant increases in plasma isoflavones with treatment for all doses compared to controls without producing any toxicity. Significant increases in serum total estradiol were observed in the $40 \mathrm{mg}$ and $60 \mathrm{mg}$ isoflavone-treated arms. However, significant increase in serum free testosterone was observed in the $60 \mathrm{mg}$ isoflavone-treated arm. Since only post-intervention tissue samples were available for staining, the difference between the treatment arms and control of percentage Ki67 staining were estimated in these samples. Compared to the control group and other treatment arms, the $40 \mathrm{mg}$ isoflavone supplemented arm had the lowest percentage of cells expressing Ki-67, although this was not statistically significant for this sample size and duration of intervention (Table 1).

\section{Summary of Evidence}

We concluded that $40 \mathrm{mg}$ of purified isoflavones may be the best dose to be used in a future definitive, larger Phase II clinical trial to evaluate purified isoflavones in prostate carcinogenesis. With prolonged consistent administration of purified isoflavones, we could potentially delay onset of the disease by interfering with the later stages of prostate carcinogenesis or growth and progression of preneoplastic and histologic cancer. Based on the promising trends observed in our study and that of others, including the relative safety compared to agents currently available for $\mathrm{CaP}$ chemoprevention [4244], a definitive Phase II clinical trial powered to examine the effects of purified isoflavones in stabilizing or inhibiting the progression of $\mathrm{CaP}$ in larger cohorts diagnosed with early stage $\mathrm{CaP}$ was the next logical step.

\section{Study Design}

Our broad long-term goal is to develop non-toxic agents that can be consumed safely over long periods to prevent progression of $\mathrm{CaP}$ in men at high risk, using validated markers to monitor changes in disease progression. This 5-year project is a randomized double-blind placebo controlled Phase II clinical trial targeting 260 AA and Caucasian men between ages 30 and 80 , diagnosed with clinically localized $\mathrm{CaP}$. The goal of the study is to examine the safety, efficacy and mechanism of action of isoflavones administered daily at a dose of $40 \mathrm{mg}$ per day ( $v s$. a placebo) for 4-6 weeks. Evaluation of the intervention will focus on safety and $\mathrm{CaP}$ progression, as measured by a combination of validated biochemical, clinical, grade progression and genetic IEBs, in addition to examining and validating the molecular target of isoflavones. Cohort participants will be recruited from clinical sites at the Moffitt Cancer Center, James A. Haley Veterans Administration Hospital and The University of Florida and hospitals affiliated with the Moffitt Cancer Center forming the Moffitt Affiliate Network, specifically focusing on groups serving AA men, utilizing our community leaders and physicians. At baseline, and end of study, participants will undergo interviews and laboratory analysis for determining compliance and the absence of toxicity caused by the study agent. Subjects will have received a baseline biopsy and transrectal ultrasound that will determine eligibility to the clinical trial, using uniform established diagnostic criteria for prostate cancer and be admitted to the study on the day they make a decision to have prostatectomy. Men will be examined on the day of prostatectomy (four to six weeks (+/-3 days) from start of study agent), at which time, serum, plasma and tissue samples will be obtained for analysis of biochemical, compliance,

\begin{tabular}{|l|l|l|l|}
\hline Arm & Number of Subjects & Mean Ki-67(\%) & SD \\
\hline Isoflavones $40 \mathrm{mg}$ & 12 & $3.20^{*}$ & 2.25 \\
\hline Isoflavones $60 \mathrm{mg}$ & 11 & 4.11 & 3.52 \\
\hline Isoflavones $80 \mathrm{mg}$ & 10 & 4.63 & 2.67 \\
\hline Control & 11 & 4.22 & 1.86 \\
\hline
\end{tabular}

Table 1: Tissue Percentage Ki-67 Staining Post Intervention with Purified Isoflavones Treatment Arm. 
safety and mechanistic molecular markers planned in the study. We have established a multidisciplinary team including special teams for administration, recruitment, intervention and retention, biomarkers, and data safety monitoring who work closely together to provide efficient, standardized and centralized services for successful implementation of this proposal. Prior to study initiation, the Human Subjects Committees of each of the participating institutions approved all study procedures. Participating sites are provided a fee for subject recruitment as well as reimbursement for initial IRB submission and subsequent continuing review fees while participating. The subjects who participate do not receive reimbursement for time or travel as there are no extra visits required as part of participation in this study.

\section{Hypothesis and Objectives}

Our central hypothesis is that the effect of supplementation with a constant dose of purified isoflavones ( $v s$. a placebo) will produce a corresponding increase in plasma levels of isoflavones, resulting in modulation of serum steroid hormone levels, indicated by an increase in serum estradiol and decrease in free testosterone, thereby potentially contributing to a decrease or stabilization of disease progression in men diagnosed with localized prostate cancer as indicated by decreased surrogate marker of proliferation, serum PSA and Ki-67 expression and increased apoptosis. Based on our preliminary study [29], we hypothesize that the primary pathway by which isoflavones will suppress prostate tumorigenesis is mediated by the ER $\beta$, which can be suppressed by ER $\alpha$ in prostate cancer cells such that ER $\beta$ is decreased. In addition, genistein inhibits androgen signaling through FOXO1 by down regulating AR expression, resulting in apoptosis and leading to the suppression of prostate carcinogenesis. We additionally hypothesize that the effectiveness of isoflavones to modulate prostate carcinogenesis will be significantly higher in AA men compared to Caucasian men. We plan to test these hypothesis using the following objectives:

\section{Primary objectives}

The primary endpoints for this study are to assess the comparative safety and efficacy of administering $40 \mathrm{mgs}$ of purified isoflavones ( $v s$. a placebo) a day in AA men and Caucasian men in the pre-surgical period prior to radical prostatectomy (minimum 4 weeks, maximum 6 weeks). Safety will be evaluated by the incidence and severity of adverse events (AEs) evaluated using NCI-CTG Toxicity Criteria, comprehensive metabolic profile and complete blood counts. Efficacy will be evaluated by differences in the change in serum steroid hormones (decrease in free testosterone, IGF and increase in total estradiol) and changes in biochemical and grade progression markers such as prostate specific antigen (PSA), tumor volume, (TV), percent Ki-67 and Gleason Score (GS) between thetreatment and placebo arms as well as between AA and Caucasian men.

\section{Secondary objectives}

The secondary objectives are to determine the mechanism of action of purified isoflavones at a dose of $40 \mathrm{mg}$ per day $v s$. placebo administered to AA men and CM in the pre-surgical period prior to radical prostatectomy. This will be evaluated by the expression levels of the androgen receptor; FOXO1 and FOXO target genes; and ratio of ERa/ER $\beta$. Additionally, with the consent of participating subjects a create a specimen repository (serum and prostate biopsies) is being created and will be used to assess other genetic or environmental factors that may influence incidence and progression of prostate cancer, and to test future hypotheses related to prostate cancer, as and when possible.

\section{Target Population}

Several models of interventions with chemopreventive agents have been used in clinical trials. Since prostate cancer has been demonstrated to have a long latency period, it would be impossible to evaluate chemoprevention agents in healthy or high risk populations. Models that have been recommended and successfully utilized by our group and others for molecular-mechanism-based approaches in phase II-III clinical trials to evaluate agents for prostate cancer chemoprevention, requiring tissue from biopsy or prostate have targeted cohorts with early stage disease (watchful waiting, Gleason score $<6$ ), men diagnosed with localized $\mathrm{CaP}$ (Pre surgical) and men diagnosed with HGPIN (during surveillance). These time periods provide the best opportunity for evaluating promising agents for chemoprevention and to delineate the mechanism of action and molecular targets of specific promising agents to prevent disease progression and shifting the time of diagnosis to invasive disease. Specifically, the pre-surgical model that we use in this clinical trial provides an excellent model in phase II clinical trials to accelerate the evaluation of initial biological response to chemoprevention agents such as isoflavones, demonstrated by the modulation of validated endpoint biomarkers in prostate cancer tissue obtained during biopsy and prostatectomy. This model, in addition, reduces the burden and improves safety to the subjects by aligning the data collection (prostate tissue sample) with the timing of standard clinical/surgical protocols (4-6 weeks between biopsy and prostatectomy). Identifying mechanism of action and molecular targets of isoflavones is critical, as proposed in our study. In addition to informing future intervention trials of the comparative safety and efficacy of isoflavones, our study has the potential to define other surrogate endpoints for CaP risk, specifically in the AA cohort, thus increasing the impact of our study. However, these methods are not without risk to subject. Risk to subject includes unknown effects of exposure to study agent after diagnosis of prostate cancer. However, by using a systematic approach of evaluating the agent in preclinical and phase I clinical trials to inform the design of phase II clinical trials and meticulous safety monitoring built into the trial design, we have reduced any potential harm to subject. On the other hand, these agents and their effectiveness nor mechanism can not be evaluated in healthy or even high risk populations where a biopsy to obtain tissue markers, would present undue risk to subject.

\section{Agent Selection and Description}

The agent used in this Phase II trial is a botanical test compound developed from Novasoy $400^{\circledast}$ provided to us by Archer Daniels Midland Company, Decatur, IL 62525. Novasoy $400^{\circledR}$ is a soy-based isoflavone concentrate extracted to assure that the ratio of isoflavones as well as the aglycone and glycoside isoforms are maintained as they would be found in soybeans and unfermented soy foods. The purified isoflavones are standardized based on isoflavone content, and are maintained on a stability monitoring program to ensure that there is no reduction in active component during the period of use. They are produced under current food Good Manufacturing Practices (GMPs) and a HACCP program.

The study agent and placebo are compounded by Carrollwood Pharmacy, Tampa, Florida. For the study agent, Novasoy $400^{\circ}$ is combined with the filler Avicel PH105, a methyl cellulose blend and formulated to deliver $20 \mathrm{mg}$ aglycone equivalent isoflavones per capsule. The final product is analyzed for accuracy by Archer Daniels Midland (ADM). The placebo contains only Avicel PH105. Both are 
compounded in an opaque, gelatin capsule to conceal any differences between the active and placebo capsules. The Principal Investigator holds an investigator-held Investigational New Drug approval from the FDA for this dose and use $(\# 61,949)$ in the preliminary clinical trial.

\section{Screening, Registration and Randomization Procedures}

Potential subjects are screened by the research coordinator using an Eligibility Checklist. The study population will consist of men who meet the following eligibility criteria: (a) AA and Caucasian men between the ages of 30 (to be inclusive of AA men with earlier age diagnosis) and 80; (b) diagnosis of localized $\mathrm{CaP}$, based on pathological assessment from biopsy specimens; (c) with no prior or current therapy for $\mathrm{CaP}$ or history of cancer except non-melanoma skin cancer; (d) scheduled for prostatectomy between 4-6 weeks (+/3) days after registration; (e) No known history of hepatic or renal disease (LFTs (SGOT/SGPT) $<2.5 \mathrm{x}$ upper limit of normal, Actual creatinine clearance of $>60$ utilizing the Cockroft-Gault formula (1976), which employs creatinine measurements and a patient's weight to predict the clearance. A modification of this formula, useful for the common units of measure is as follows: $\mathrm{X}=(140$-age $) \mathrm{x}$ Weight in Kgs $\mathrm{x}$ Constant / Creatinine in $\mu \mathrm{mol} / \mathrm{L}$. The constant is 1.23 for men and 1.04 for women); (f) Omnivorous diet; (g) No evidence of prostatitis or urinary tract infection; (h) Able and willing to give written informed consent; (i) Currently not using nutritional supplements (other than the multivitamin/mineral provided for all study participants); (j) not allergic to study supplements; (k) not on antibiotics; (l) men who do not consume more than $3 \mathrm{oz}-4 \mathrm{oz}$ of soy or soy products per week; (m) willing to comply with scheduled visits as proposed for 4-6 weeks; (n) not taking steroid hormones or medications which have known impact on PSA; (o) health status cleared by primary MD or urologist and (p) ECOG performance status of $0-1$. Allowing a dropout rate of $20 \%$ as previously observed in chemoprevention trials, we plan to recruit 130 AA men and 130 Caucasian men $(n=260 ; 65 / a r m)$ to total 260 men over the study period. Once eligibility is determined, subjects are then provided with information about the research study and informed of the experimental nature of therapy, alternatives, potential benefits, sideeffects, risks and discomforts. Interested subjects who appear eligible based on information available at this time are given the opportunity to sign an Informed Consent document.

All subjects who sign an Informed Consent document are assigned a registration number assigned by the clinic site. As subjects are registered, demographic information and registration information are entered into a computerized system for tracking purposes. Participating subjects have blood drawn for confirmation of eligibility, if not available from within the last fourteen days (e.g., to verify normal liver function). Subjects are provided with: (a) an information package regarding the study; (b) lists of foods, medications and nutritional supplements to avoid during the study period; (c) log for recording study agent intake (which includes assessment for AEs and concomitant medications); (d) two-day diet recall forms with instructions; (e) Godin-Leisure Time Exercise questionnaire.

There is no run-in period for this study. Only men meeting all inclusion and exclusion criteria are randomized to a treatment or placebo arm using the Moffitt SRARS program, a web delivered application that records subject registrations and provides blocked randomization assignments. A protocol is developed in the SRARS program using randomization lists, stratified by race (AA and other) and by site was developed by the Moffitt Statistics department, to ensure that for every site with subjects in the 2 racial groups, a corresponding placebo and treatment assignment is planned. Descriptive summary statistics will be reported on patient characteristics at baseline (epidemiological data including demographics, personal and physical characteristics, family history, history of environmental and personal exposures, alcohol intake, dietary and physical activity, cancer screening, medication use including nutritional supplements and dietary intake, and several plasma and serum biomarker levels) as well as outcome variables at 4-6 weeks post intervention, respectively, both by arm and race. An attempt will be made to discern any possible differences in the baseline patient characteristics between the two arms within each race group. This will hopefully identify any imbalances that may be large enough but have occurred at randomization by chance that will need to be controlled for in the statistical analyses in order not to confound the study results and their interpretations. All baseline group comparisons of demographic, anthropometrics, clinical, and dietary measures will be conducted empirically without using formal statistical tests.

As this is a double-blinded study, the treatment/randomization is hidden from all users except the pharmacist preparing and labeling the treatment drug. Once the randomization is complete, the study agent is mailed overnight via UPS. If lab results disqualify from participation, subjects are notified of results and taken off study. They are also given an opportunity to speak with the study physician, a copy of their labs made available to them and the samples collected during screening are destroyed.

At the end of the study, on the day of prostatectomy, participants undergo interviews and serum, plasma and tissue samples are obtained. Laboratory analysis is completed for safety (the determination of and the absence of toxicity caused by the study agent) and compliance, as well as evaluation of the biochemical and mechanistic molecular markers considered in this study (Scheme 1: Study Schema).

Phase II Clinical Trial of Purified Isoflavones in Prostate Cancer: Comparing Safety, Effectiveness and Mechanism of Action between African American and Caucasian Men.

African American (AA) and Caucasian men between the ages of 30 (to be inclusive of AA men with earlier age diagnosis) and 80 with a diagnosis of localized $\mathrm{CaP}$, based on pathological assessment from biopsy specimens;

with no prior or current therapy for $\mathrm{CaP}$ or history of cancer except non-melanoma skin cancer;

scheduled for prostatectomy between 4-6 weeks (+/-3 days) after start of study agent $(n=260)$

\section{$\downarrow$}

Screening/Randomization: Informed consent; confirm diagnostic prostate biopsy pathology; Digital Rectal Exam (DRE); Prostate Specific Antigen (PSA); (DRE and PSA do not need to be repeated if results are available from pre-biopsy tests); Comprehensive Metabolic Panel (CMP) including LFTs; Complete Blood Count (CBC) (these do not need to be completed if results available from within 14 days prior to randomization); serum steroid hormones; collect plasma for baseline isoflavone measurements; collect buffy coat for banking; obtain anthropometric measurements (weight, height, BMI, waist and hip circumference); On-study Epidemiological Questionnaire; assess baseline Lower Urinary Tract Symptoms (LUTS) score; signs and symptoms assessment; concomitant medication assessment; distribute and collect GodinLeisure Time Exercise Questionnaire; distribute instructions and 
forms for 2-day Diet Recall; obtain tissue from diagnostic biopsy for baseline measurements; randomize to active agent $(40 \mathrm{mg}$ purified isoflavones daily) or placebo $(\mathrm{n}=65 / \mathrm{arm})$

\section{$\downarrow$}

Intervention: Confirm eligibility based on screening tests; self administration of purified isoflavones ( $40 \mathrm{mg}$ daily) or placebo for 4-6 weeks; weekly telephone contact to assess signs and symptoms and concomitant medications while on study

\section{$\downarrow$}

End of Intervention (Week 6 or time of Prostatectomy): Prostate Specific Antigen (PSA); Comprehensive Metabolic Panel (CMP) including LFTs; Complete Blood Count (CBC); serum steroid hormones; collect plasma for isoflavone measurements; collect buffy coat for banking; obtain anthropometric measurements (weight, height, BMI, waist and hip circumference); collect and review 2-day Diet Recall forms; signs and symptom assessment; concomitant medication assessment; review study agent intake log and check compliance (pill count); assess end of intervention Lower Urinary Tract Symptoms (LUTS); distribute and collect Godin-Leisure Time Exercise Questionnaire

\section{$\downarrow$}

Post-intervention Follow-up ( 7 days (+/-3 days) post-treatment): Telephone contact to assess signs and symptoms and concomitant medications occurring post-treatment

Endpoints:

Efficacy (Primary): Change in percent Ki-67 evaluated in prostate cancer tissue specimens after 4-6 weeks of intervention with purified isoflavones (40 $\mathrm{mg}$ daily) vs. Placebo.

Safety (Primary): Incidence and severity of AEs occurring during intervention with either $20 \mathrm{mg}$ purified isoflavones bid or placebo.

Secondary (When tissue available):

$\rightarrow$ Drug effect measurements in tissue samples

$\rightarrow$ To determine if purified isoflavones (40 mg daily) vs. placebo results in decreased expression of the androgen receptor and increased expression levels of FOXO1 and its target genes from baseline to prostatectomy in prostate cancer patients

$\rightarrow$ Whether the treatment effect of the study agent in the ARFOXO axis is more profound in AA patients as compared to the Caucasian group and correlates inversely with $E R \alpha / E R \beta$ ratio

\section{Adherence/Compliance During Intervention}

During study participation, subjects are to self administer one capsule of study agent daily each morning and evening, with meals. In addition to taking the study agent, subjects are instructed to take one multivitamin/mineral daily. Subjects are also asked to complete diet recall and exercise questionnaires at specified time periods, avoid consumption of foods that are rich in isoflavones and avoid herbs and nutritional supplements containing isoflavones; complete daily study agent intake logs, record concomitant medications, and any signs or symptoms. The goal is to maintain $\geq 85 \%$ compliance with study agent intake. Subjects are contacted weekly via telephone from the time of consent until one week following their surgery.

\section{Safety Monitoring}

All subjects are assessed clinically for toxicities prior to randomization and at the end of intervention. Baseline and end of study lab work for safety includes complete blood count (CBC) and comprehensive metabolic profile (CMP), including liver function tests (LFTs). Study agent intake logs are used to assess signs/symptoms and concomitant medications and are reviewed weekly during the telephone call and at the end of the study. Compliance with study agent intake is measured via pill counts, completed at the end of the study. Any toxicities (adverse events) occurring during the investigation are reviewed by the treating physician and managed according to standard medical practice.

\section{Biomarkers of Disease Progression}

Biomarkers of disease progression will include serum steroid hormones, PSA, apoptotic index, Tumor Volume (TV), Gleason Score (GS); Symptoms including Lower Urinary Tract Symptoms (LUTS). Diagnostic Prostate Specific Antigen (PSA) will be measured at baseline and end of study as surrogate markers of tumor progression. The serum PSA will be determined using Bayer Centaur Chemluminescene by Quest laboratories. Serum PSA, although well established as a biomarker of $\mathrm{CaP}$, is not specific to neoplasia. The literature does not currently support that the level is directly related to the degree of neoplastic progression. The validation of PSA as an intermediate biomarker awaits further data, some of which may be obtained from the large NCI Prostate, Lung, Colorectal \& Ovarian Cancer Screening Trial in which PSA is being monitored over several years in over 30,000 men [45]. Traditionally, clinicians have relied on PSA as a prognostic indicator in addition to stage, tumor grade and volume. Because the serum level of PSA is proportional to the volume of tumor present, PSA has become an integral part of disease management in this population. The growth of CaP is exponential at all stages. Thus the doubling of the serum PSA will reflect the biological doubling rate of tumor. Thus, PSA continues to be a valuable surrogate marker offering a dynamic view of disease progression and possibly a marker of chemoprevention efficacy, specifically in AA men [46].

Confirmation of a diagnosis of $\mathrm{CaP}$ in prostate biopsy samples will be determined by the site pathologist at each participating institution and Gleason Score determined. Prostate tissue will be obtained by the surgeons at baseline and at post-intervention with isoflavones at prostatectomy. Representative slides from the initial pre-interventional biopsy and from the post-interventional prostatectomy are required for analysis. At each site, all patients included in this study will have formalin fixed paraffin-embedded prostatic biopsies that are initially sectioned and stained with hematoxylin and eosin ( $\mathrm{H} \& \mathrm{E})$. At each site, the site pathologist will evaluate the $\mathrm{H} \& \mathrm{E}$ stained sections by light microscopy for the presence of malignancy, using the diagnostic criteria currently utilized and described by the AUA [47]. Gleason score will also be centrally verified by the primary site Chair of Pathology. Immunohistochemical Staining for Ki-67 Nuclear Antigen and Image Cytometric Determination of Apoptosis by the TUNEL Method: Expression of Ki-67 has been shown to be clearly different in normal, HGPIN, and CaP biopsy specimens. Multiple studies have demonstrated that mean proliferation indices, as measured by Ki-67 staining, are associated with disease progression, stage, Gleason score, and mean pretreatment prostate-specific antigen level and show an 
increase from benign to malignant tissue. Ki-67 is thus considered a biomarker that can be utilized in clinical trials that compare biopsy specimens over time, biopsy and radical prostatectomy (RP) specimens (i.e., neoadjuvant studies), or multiple biopsy specimens in the setting of chemoprevention [48]. We have thus identified Ki-67 as the primary marker to study effectiveness of intervention with isoflavones.

The following steroid hormone will be measured at baseline and end of study: Sex Hormone Binding Globulin (SHBG), Total \& Free Testosterone, Estradiol, IGF I and IGF Binding Protein -3 (IGFBP3) by Quest Labs [Extraction, chromatography, radioimmunoassay (RIA)]. Although the mechanism of action of isoflavones is not clear, it has been observed that steroid hormones play an important role in increasing or decreasing the risk of prostate cancer. Patients with prostate cancer have been observed to have higher free testosterone (unbound) levels and lower levels of sex-hormone binding globulin (SHBG), estrone and estradiol. Androgens are essential for the function and growth of the prostate and are known to stimulate the proliferation of human prostatic cells. Administration of hormonal therapies has been shown to produce prostate cancer in rodents, while castration and estrogen therapy can reduce the risk of prostate cancer. It is clear from recent studies that testosterone and estradiol are important contributors of androgenic and estrogenic activity. Furthermore, sex-hormone binding globulin, because it binds to and sequesters testosterone and estradiol, controls the bioavailability of these sex hormones to target cells as well as their mutual balance. This effect on the active estrogen/testosterone balance may be another potential autoregulatory mechanism for the protective effect of SHBG in prostate cancer. Recent evidence suggests that SHBG can function as a hormone with a direct interaction with prostate cells. The insulinlike growth factor type I receptor (IGF-IR) has been suggested to play an important role in prostate cancer progression and possibly in the progression to androgen-independent (AI) disease. Androgens upregulate insulin-like growth factor-I receptor (IGF-IR) expression and sensitize prostate cancer cells to the biological effects of IGF-I. IGF-1 levels increase and IGFBP3 protein levels decrease during the progression of prostate cancer and thus selected to be an additional indicator of modulation by isoflavones [34,39,49-50].

\section{Markers of Mechanism}

We plan to determine if purified isoflavones at a dose of $40 \mathrm{mg}$ daily (20 $\mathrm{mg}$ bid) $v s$. placebo results in decreased expression of the androgen receptor and increased expression levels of FOXO1 and its target genes from baseline to prostatectomy in prostate cancer patients and whether the treatment effect of the study agent in the AR-FOXO axis is more profound in AA patients as compared to the Caucasian group and correlates inversely with ER $\alpha / E R \beta$ ratio. Prostatectomy samples of $\mathrm{CaP}$ lesion and normal adjoining tissues will be separated into two parts. The first part will be fixed and paraffin embedded for immunohistochemistry in the Moffitt Pathology Immunohistochemistry laboratory or by local pathologist to determine the expression/ localization of FOXO factors. The second part will be snap-frozen for molecular analysis, including RNA isolation for RT-PCR analyses of FOXO target genes to address the mechanisms of isoflavones.

\section{Treatment Interruption or Termination}

There are no reductions in the purified isoflavone dose during this study and no suspension of agent required for AEs graded 1-2. Study drug will be permanently discontinued for grade 3 and 4 AEs, unless clearly not attributable to therapy as determined by the study team.
All AE information is captured according to standard procedures and evaluated using CTCAE Term (v 4.0). Subjects who have had an interruption of intake of more than 7 calendar days due to AE may be withdrawn from the study at the discretion of the investigator. Subjects who have an unresolved AE at the time of withdrawal from study treatment are followed until resolution of the event, if possible. If an AE persists for more than 30 days after a subject goes off study, the subject is referred to his personal physician.

\section{Statistical Analysis}

The primary endpoint is the percentage Ki-67 expression measured post intervention at prostatectomy. It is a mean proliferation index associated with disease progression, stage, Gleason score, and pretreatment PSA level and general increases from benign to malignant tissue. We have thus identified Ki-67 as the primary marker to study effectiveness of intervention with soy isoflavones. Secondary endpoints include several biomarkers such as serum steroid hormones, PSA, apoptotic index, levels of AR, FOXO1 and FOXO target genes, and ratio of $\mathrm{ER} \alpha / \operatorname{Er} \beta$, measured both at baseline and post intervention at prostatectomy, as well as tumor volume (TV), Gleason Score (GS), Lower Urinary Tract Symptoms (LUTS), standard pill counts, diet records and plasma isoflavones. The changes in biomarkers will be analyzed as continuous variables as appropriate. Both absolute and relative changes will be studied for a continuous outcome variable unless otherwise specified. Normality assumptions will be verified and the log transformation implemented, if appropriate, for any continuous outcome variable.

The sample size/power calculations are based on the primary endpoint percent Ki-67 level post intervention. Based on limited data available from previous studies and our own preliminary research, we expect that the mean and standard deviation (SD) of percent Ki-67 post intervention for our population in each of the four arms will be as described in Table 2. That is, due to the AA patient population being slightly younger and having more aggressive disease than Caucasian men, their Ki-67 level in the placebo group is expected to be $1 \%$ higher on average compared to the Caucasian control group. In the meantime, the treatment effect for the AA population is projected to be greater than that for the Caucasian population. In fact, the above table assumes a $24 \%$ difference between treatment and control arms for Caucasian men while it is $36 \%$ for the AA men.

Thus, 52 evaluable subjects in each arm will yield at least $80 \%$ of power at a two-sided alpha level of 0.10 for detecting a group difference of $24 \%$ or more in the average Ki-67 post intervention between the treatment and control arms in Caucasian group. The power will increase to $92 \%$ for AA group if the difference remains at $24 \%$ (control vs. treated: 5.22 vs. 3.97). If, however, our projected difference is realized, i.e., the difference is at $36 \%$ as in the above table, the power will be at least $99 \%$ for AA group. To take into account a potential $20 \%$ drop-out rate as previously observed in chemoprevention trials for this study, we plan to recruit and randomize $65 \times 4=260$ men diagnosed with clinically localized $\mathrm{CaP}$ into this trial in order to ensure that there will be at least 52 evaluable subjects in each arm for adequate statistical power in terms of the primary study endpoint.

\begin{tabular}{|l|l|l|}
\hline Mean (SD) of Ki-67 & Caucasian & AA \\
\hline Treatment & $3.20(2.25)$ & $3.34(2.25)$ \\
\hline Control & $4.22(1.86)$ & $5.22(1.86)$ \\
\hline
\end{tabular}

Table 2: Anticipated mean and standard deviation (SD) of percent Ki-67 post intervention. 
Citation: Kumar N, Crocker T, Smith T, Pow-Sang J, Spiess PE, et al. (2012) Prostate Cancer Chemoprevention Targeting High Risk Populations: Model for Trial Design and Outcome Measures. J Cancer Sci Ther S3:007. doi:10.4172/1948-5956.S3-007

Page 9 of 10

\section{Data Management and Study Monitoring}

The Data Management team consists of the PI, Research IT core analyst, Chief Biostatistician, Responsible research project coordinator and Data Manager at the MCC, Tampa, FL. All collected data is entered from source documents or case report forms (CRF's) directly into the web-based ONCORE system by the data manager. Toxicities are monitored continuously through the trial by the PI and study physician at each site. Additionally, the study team is guided by a Data Safety Monitoring Board (DSMB) and an External Data and Safety Monitoring Board (EDSMB). Statistical analyses will be performed and reviewed by the EDSMB once fifty patients have completed the entire 4-6 week study period. The EDSMB will review the accumulating outcome data and evaluate the results in the context of results of any other trials that might be reported in the interval. The DSMB will, in addition, recommend if any information regarding adverse events must be released to subjects and make any decisions about recommending external review, as needed. The EDSMB can recommend early termination if a serious imbalance occurs and the estimated risk of harm appeared to warrant such action.

\section{Future Directions}

Purified Isoflavones in Prostate Cancer is a Phase II, Randomized, Double-blind, Multi-centered Study, currently ongoing at three clinical sites across the United States. The goal of this study is to evaluate the efficacy, safety and mechanism of action of $40 \mathrm{mg}$ of purified isoflavones $v s$. placebo, with a comparison between AA and Caucasian men. The pre-surgical model that we use in this clinical trial provides an excellent model for Phase II clinical trials to accelerate the evaluation of initial biological response to chemoprevention agents such as isoflavones, demonstrated by the modulation of validated endpoint biomarkers in prostate cancer tissue obtained during biopsy and prostatectomy. In addition, this model reduces the burden and while prioritizing safety to the subjects by aligning the data collection (prostate tissue sample) with the timing of standard clinical/surgical protocols (4-6 weeks between biopsy and prostatectomy). Future chemoprevention trials may utilize this approach to truly move research from the bench to the bedside, where it can have the greatest impact on addressing health disparities.

Identifying the mechanism of action and molecular targets of isoflavones is critical. In addition to informing future intervention trials of the comparative safety and efficacy of isoflavones, our study has the potential to define other surrogate endpoints for $\mathrm{CaP}$ risk, specifically in the AA cohort, thus increasing the impact of our study. Once these studies are completed and the comparative efficacy, safety and mechanism of action are established, this can lead to the development and implementation of focused, larger Phase III trials that can specifically target high risk populations, including AA men who are at relatively higher risk and mortality for this disease than any other population, thus contributing to the elimination of health disparities.

\section{Acknowledgement}

The research study was funded by the National Institute of Health-1 P20 MD003375-01.

\section{References}

1. Cancer Facts and Figures (2011) Atlanta, GA: American Cancer Society

2. Holmberg L, Bill-Axelson A, Garmo H, Palmgren J, Norlén BJ, et al. (2006) Prognostic markers under watchful waiting and radical prostatectomy. Hematol Oncol Clin North Am 20: 845-855

3. Johansson JE, Andrén O, Andersson SO, Dickman PW, Holmberg L, et al
(2004) Natural history of early, localized prostate cancer. JAMA 291: 27132719 .

4. Kelloff GJ, Lieberman R, Steele VE, Boone CW, Lubet RA, et al. (1999) Chemoprevention of prostate cancer: concepts and strategies. Eur Urol 35 342-350.

5. Addressing Racial and Ethnic Healthcare Disparities. Where do we go from here? Washington, DC, National Academy Press, 2008.

6. Kumar NB, Yu D, Akinremi TO, Odedina FT (2008) Comparing dietary and other lifestyle factors among immigrant Nigerian men living in the US and indigenous men from Nigeria: potential implications for prostate cancer risk reduction. J Immigr Minor Health 11: 391-399.

7. Adlercreutz H, Honjo H, Higashi A, Fotsis T, Hämäläinen E, et al. (1991) Urinary excretion of lignans and isoflavonoid phytoestrogens in Japanese men and women consuming a traditional Japanese diet. Am J Clin Nutr 54: 1093-1100.

8. Blumenfeld AJ, Fleshner N, Casselman B, Trachtenberg J (2000) Nutritiona aspects of prostate cancer: a review. Can J Urol 7: 927-935.

9. Brössner C, Petritsch K, Fink K, Auprich M, Madersbacher S, et al. (2004) Phytoestrogen tissue levels in benign prostatic hyperplasia and prostate cancer and their association with prostatic diseases. Urology 64: 707-711.

10. Chan JM, Gann PH, Giovannucci EL (2005) Role of diet in prostate cancer development and progression. J Clin Oncol 23: 8152-8160.

11. Morton MS, Chan PS, Cheng C, Blacklock N, Matos-Ferreira A, et al. (1997) Lignans and isoflavonoids in plasma and prostatic fluid in men: samples from Portugal, Hong Kong, and the United Kingdom. The Prostate 32: 122-128.

12. Shimizu H, Ross RK, Bernstein L, Yatani R, Henderson BE, et al. (1991) Cancers of the prostate and breast among Japanese and white immigrants in Los Angeles County. Br J Cancer 63: 963-966.

13. Bemis DL, Capodice JL, Desai M, Buttyan R, Katz AE (2004) A concentrated aglycone isoflavone preparation (GCP) that demonstrates potent anti-prostate cancer activity in vitro and in vivo. Clin Cancer Res 10: 5282-5292.

14. Fotsis T, Pepper M, Adlercreutz H, Hase T, Montesano R, et al. (1995) Genistein, a dietary ingested isoflavonoid, inhibits cell proliferation and in vitro angiogenesis. J Nutr 125: 790S-797S.

15. Jonnalagadda SS, Mitchell DC, Smiciklas-Wright $\mathrm{H}$, Meaker KB, Van Heel N, et al. (2000) Accuracy of energy intake data estimated by a multiple-pass, 24-hour dietary recall technique. J Am Diet Assoc100: 303-308.

16. Messina M, Barnes S (1991) The role of soy products in reducing risk of cancer J Natl Cancer Inst 83: 541-546.

17. Ouchi $\mathrm{H}$, Ishiguro $\mathrm{H}$, Ikeda N, Hori M, Kubota $Y$, et al. (2005) Genistein induces cell growth inhibition in prostate cancer through the suppression of telomerase activity. Int J Urol 12: 73-80.

18. Adlercreutz H, Mousavi Y, Clark J, Höckerstedt K, Hämäläinen E, et al. (1992) Dietary phytoestrogens and cancer: in vitro and in vivo studies. J Steroid Biochem Mol Biol 41: 331-337.

19. Skogseth H, Larsson E, Halgunset J (2006) The invasive behaviour of prostatic cancer cells is suppressed by inhibitors of tyrosine kinase. APMIS 114: 61-66.

20. Huang X, Chen S, Xu L, Liu Y, Deb DK, et al. (2005) Genistein inhibits p38 map kinase activation, matrix metalloproteinase type 2 , and cell invasion in human prostate epithelial cells. Cancer Res 65: 3470-3478.

21. Li Y, Kucuk O, Hussain M, Abrams J, Cher ML, et al. (2006) Antitumor and antimetastatic activities of docetaxel are enhanced by genistein through regulation of osteoprotegerin/receptor activator of nuclear factor-kappaB (RANK)/RANK ligand/MMP-9 signaling in prostate cancer. Cancer Res 66 : 4816-4825.

22. Handayani R, Rice L, Cui Y, Medrano TA, Samedi VG, et al. (2006) Soy isoflavones alter expression of genes associated with cancer progression, including interleukin-8, in androgen-independent PC-3 human prostate cancer cells. J Nutr 136: 75-82.

23. Raschke M, Rowland IR, Magee PJ, Pool-Zobel BL (2006) Genistein protects prostate cells against hydrogen peroxide-induced DNA damage and induces 
Citation: Kumar N, Crocker T, Smith T, Pow-Sang J, Spiess PE, et al. (2012) Prostate Cancer Chemoprevention Targeting High Risk Populations: Model for Trial Design and Outcome Measures. J Cancer Sci Ther S3:007. doi:10.4172/1948-5956.S3-007

expression of genes involved in the defence against oxidative stress. Carcinogenesis 27: 2322-2330

24. Kazi A, Daniel KG, Smith DM, Kumar NB, Dou QP (2003) Inhibition of the proteasome activity, a novel mechanism associated with the tumor cell apoptosis-inducing ability of genistein. Biochemical pharmacology 66: 965-976.

25. Kazi A, Smith DM, Daniel K, Zhong S, Gupta P, et al. (2002) Potential molecular targets of tea polyphenols in human tumor cells: significance in cancer prevention. In vivo 16: 397-403.

26. Nam S, Smith DM, Dou QP (2001) Ester bond-containing tea polyphenols potently inhibit proteasome activity in vitro and in vivo. J Biol Chem 276: 13322 13330

27. Katoh M (2004) Human FOX gene family (Review). Int J Oncol 25: 1495-1500.

28. Busby MG, Jeffcoat AR, Bloedon LT, Koch MA, Black T, et al. (2002) Clinical characteristics and pharmacokinetics of purified soy isoflavones: single-dose administration to healthy men. Am J Clin Nutr 75: 126-136.

29. Li P, Lee H, Guo S, Unterman TG, Jenster G, et al. (2003) AKT-independent protection of prostate cancer cells from apoptosis mediated through complex formation between the androgen receptor and FKHR. Mol Cell Biol 23: 104-

30. Huang H, Muddiman DC, Tindall DJ (2004) Androgens negatively regulate forkhead transcription factor FKHR (FOXO1) through a proteolytic mechanism in prostate cancer cells. J Biol Chem 279: 13866-13877.

31. Davis JN, Kucuk O, Sarkar FH (2002) Expression of prostate-specific antigen is transcriptionally regulated by genistein in prostate cancer cells. Mol Carcinog 34: 91-101.

32. Nelson KA, Witte JS (2002) Androgen receptor CAG repeats and prostate cancer. Am J Epidemiol 155: 883-890.

33. Fischer L, Mahoney C, Jeffcoat AR, Koch MA, Thomas BE, et al. (2004) Clinica characteristics and pharmacokinetics of purified soy isoflavones: multiple-dose administration to men with prostate neoplasia. Nutr Cancer 48: 160-170.

34. Kumar NB, Krischer JP, Allen K, Riccardi D, Besterman-Dahan K, et al. (2007) A Phase II randomized, placebo-controlled clinical trial of purified isoflavones in modulating steroid hormones in men diagnosed with localized prostate cancer. Nutr Cancer 59: 163-168.

35. Dalais FS, Meliala A, Wattanapenpaiboon N, Frydenberg M, Suter DA, et al (2004) Effects of a diet rich in phytoestrogens on prostate-specific antigen and sex hormones in men diagnosed with prostate cancer. Urology 64: 510-515.

36. deVere White RW, Hackman RM, Soares SE, Beckett LA, Li Y, et al. (2004) Effects of a genistein-rich extract on PSA levels in men with a history of prostate cancer. Urology 63: 259-263.

37. Kranse R, Dagnelie PC, van Kemenade MC, de Jong FH, Blom JH, et al. (2005) Dietary intervention in prostate cancer patients: PSA response in a randomized double-blind placebo-controlled study. Int J Cancer 113: 835-840.

38. Kumar NB, Cantor A, Allen K, Riccardi D, Besterman-Dahan K, et al. (2004) The specific role of isoflavones in reducing prostate cancer risk. The Prostate 59: 141-147.

39. Kumar NB, Krischer JP, Allen K, Riccardi D, Besterman-Dahan K, et al. (2007) Safety of purified isoflavones in men with clinically localized prostate cancer. Nutr Cancer 59: 169-175.

40. Schröder FH, van der Cruijsen-Koeter I, de Koning HJ, Vis AN, Hoedemaeke $\mathrm{RF}$, et al. (2000) Prostate cancer detection at low prostate specific antigen. J Urol 163: 806-812.

41. Kumar NB, Besterman-Dahan K, Kang L, Pow-Sang J, Xu P, et al. (2008) Results of a Randomized Clinical Trial of the Action of Several Doses of Lycopene in Localized Prostate Cancer: Administration Prior to Radical Prostatectomy. Clin Med Urol 1: 1-14.

This article was originally published in a special issue, Cance

Chemoprevention handled by Editor(s). Dr. Rajendra Sharma, University of North Texas, USA
42. Adams KF, Chen C, Newton KM, Potter JD, Lampe JW (2004) Soy isoflavones do not modulate prostate-specific antigen concentrations in older men in a randomized controlled trial. Cancer Epidemiol Biomarkers Prev 13: 644-648.

43. Papandreou CN, Daliani DD, Nix D, Yang H, Madden T, et al. (2004) Phase I trial of the proteasome inhibitor bortezomib in patients with advanced solid tumors with observations in androgen-independent prostate cancer. $\mathrm{J}$ Clin Oncol 22: 2108-2121.

44. Papandreou CN, Logothetis CJ (2004) Bortezomib as a potential treatment for prostate cancer. Cancer Res 64: 5036-5043.

45. Thompson IM, Ankerst DP, Chi C, Goodman PJ, Tangen CM, et al. (2006) Assessing prostate cancer risk: results from the Prostate Cancer Prevention Trial. J Natl Cancer Inst 98: 529-534.

46. Latchamsetty KC, Kim J, Porter CR (2006) Prostate specific antigen remains an independent predictor of cancer at prostate biopsy in black american men but not in white men: results from a consecutive series of 914 men. J Urol 175 : 913-917.

47. Bostwick DG, Burke HB, Djakiew D, Euling S, Ho SM, et al. (2004) Human prostate cancer risk factors. Cancer 101: 2371-2490.

48. Mucci NR, Rubin MA, Strawderman MS, Montie JE, Smith DC, et al. (2000) Expression of nuclear antigen $\mathrm{Ki}-67$ in prostate cancer needle biopsy and radical prostatectomy specimens. J Natl Cancer Inst 92: 1941-1942.

49. Wu JD, Haugk K, Woodke L, Nelson P, Coleman I, et al. (2006) Interaction of IGF signaling and the androgen receptor in prostate cancer progression. J Cell Biochem 99: 392-401.

50. Pandini G, Mineo R, Frasca F, Roberts CT Jr, Marcelli M, et al. (2005) Androgens up-regulate the insulin-like growth factor-I receptor in prostate cancer cells. Cancer Res 65: 1849-1857. 